\title{
Association of different blood parameters with the early patency of Infarct related artery in ST elevation Myocardial Infarction, a comparative analysis.
}

\author{
Reeju Manandhar, Chaofeng Sun
}

Department of cardiology, 1st affiliated hospital, Xian Jiaotong University, PR China

Corresponding author: Reeju Manandhar, Department of cardiology, 1st Affiliated hospital, Xian Jiaotong University, China, Email address: reejuman@gmail.com

\section{Abstract}

\begin{abstract}
Background and Aims: ST-segment elevation myocardial infarction(STEMI), a severe form of coronary heart disease signifies total occlusion of a coronary artery leading to myocardial necrosis, however about $10-20 \%$ of patient's presents with a patent infarct-related artery on the initial angiogram. The availability of reliable markers for prediction of patency in infarct-related artery is very limited.

Methods: We conducted a single centered retrospective study of STEMI patients undergoing primary percutaneous coronary intervention, to determine how various factors such as blood parameters differ in patients with patent infarct related artery to patients with total occlusion at the time of initial angiography done within 24hours of onset of symptoms and how they affect in outcome of the disease.

Results: Mean Platelets Volume level (11.96 fL vs. $10.92 \mathrm{fL}, \mathrm{p}<0.001)$, Lp (a) level (179.57 nmol/l vs $141.16 \mathrm{nmol} / \mathrm{l}, \mathrm{p}<0.001)$, CK-MB (290.2 vs. 190.98, $\mathrm{p}<0.001)$, total cholesterol level $(4.11 \mathrm{mmol} / \mathrm{L}$ vs. $3.8 \mathrm{mmol} / \mathrm{L}, \mathrm{p}<0.02)$ in total occlusion group were higher than in the patent infarct related artery group. Wall motion abnormality was $77.2 \%$ for 203 patients with total occlusion group and $54.2 \%$ for 83 patients with patent infarct related artery group $(\mathrm{p}<0.01)$. Mean hospital stay days were higher in total occlusion group as compared to the patent infarct related artery group $(\mathrm{p}<0.01)$.

Conclusions: Mean Platelet Volume, Lipoprotien(a), Total cholesterol, and CK-MB levels may play role in predicting patency of Infarct related artery in STEMI.
\end{abstract}

Key words: Infarct related artery; total occlusion; patency; STEMI.

\section{Introduction}

Heart disease accounts for the single most frequent cause of morbidity and mortality worldwide. Over seven million people die every year from coronary heart disease, accounting for about $12 \%$ of all deaths. ${ }^{1} \mathrm{ST}$ segment elevation on electrocardiogram, signifies total occlusion of a coronary artery leading to myocardial necrosis, however about $10-20 \%$ of patients with ST elevation Myocardial Infarction (STEMI) presents with a patent infarct-related artery (IRA) on the initial angiogram ${ }^{2}$ and the prognosis of these patients is significantly better, such as smaller size of infarction, lower incidence of heart failure, better prognosis and improved early as well as late survival when compared to that with total occlusion ${ }^{3}$. The aim of our study is to determine whether patients presenting with higher coronary flow and patent IRA at the time of initial angiography differ from patients with total occlusion in terms of various factors such as inflammatory and lipid markers and routine blood parameters and outcome of the disease.

\section{Methods}

It was a single centered study of STEMI patients undergoing Primary Percutaneous coronary intervention (PCI) within 24 hours of onset of symptoms, conducted at the Department of Cardiology, 1st Affiliated Hospital of Xi'an Jiaotong University, Xian, PR China, for 5 years duration (1st January 2008 to 31st December 2013). Ethical approval for the study was obtained from the ethics committee of Xi'an Jiaotong University. It was retrospective comparative analysis of two study groups.

Total of 871 patients underwent primary PCI at the department of Cardiology, 1st Affiliated Hospital of Xi'an Jiaotong University, Shaanxi Province, PR China, from 1st January 2008 to 31 st December 2013. Among them, 584 patients met our exclusion criteria such as: documented history of coronary artery disease, documented history of more than $70 \%$ stenosis on angiogram of IRA, chronic systemic illness and other comorbid conditions; patients admitted to the hospital 
after 24 hours period from the onset of symptoms; patients undergoing thrombolysis before coronary angiogram and patients with limited data available. Among the excluded patients, 396 patients underwent thrombolysis before coronary angiogram, 23 patients had documented history of coronary artery disease(as these patients have high likelihood of getting total occlusion even with minimum insult), 27 patients were suffering from chronic systemic illness and other comorbid conditions (Multiple organ failure-16, End-stage renal disease- 8, Lung Cancer with metastasis with AIDS- 1 , Liver cirrhosis with stroke1, Gastric cancer with chronic renal failure-1); (as their blood parameters may get influenced by their underlying disorders), 39 patients underwent coronary angiogram after more than 24 hours from onset of symptoms and 99 patients were excluded because of incomplete data.

Remaining 287 patients were then divided into two study groups on the basis of TIMI flow grade.

Group A ( $\mathrm{n}=203)$, total occlusion group, included patients with TIMI flow $\leq 1$; Group B $(n=83)$, patent IRA group, included those with TIMI flow $\geq 2$.

Data analysis was performed using SPSS 20 statistical package. Descriptive analysis of numerical data was expressed as mean, standard deviation and range, test of Normality was conducted within the groups. Variation between the two study groups were analyzed using $t$ test or Mann Whitney $U$ test depending upon the normality test. In case of categorical variables, they were expressed as frequency and percentage. Categorical data was analyzed using chi-square test. If the p value was $<0.05$ then it was considered statistically significant.

\section{Results}

Our study included 203 patients in total occlusion and 84 patients in IRA groups. Patient's descriptive and demographic characteristics are summarized in table 1 .

\section{Risk factors of Coronary Artery Disease (CAD)}

Among the traditional risk factors of CAD, prevalence of dyslipidemia was higher in total occlusion group $(14.3 \%)$ as compared to that in patent IRA group (2.4\%), which was, statistically significant $(\mathrm{P}<0.01$, Table 1 ). Prevalence of hypertension was relatively higher in total occlusion group as compared to IRA group, but the difference was not statistically significant (Table 1). Smoking was also more prevalent in total occlusion group but the difference was statistically non significant (Table 1). Diabetes was found to be more prevalent in patent IRA group than that in total occlusion group, but the difference was not significant statistically(Table 1). Family history of CAD was higher in patent IRA group as compared to that in total occlusion group but it was statistically non significant ( Table 1).

\section{Blood parameters}

Mean Platelet Volume(MPV) level was significantly higher in total occlusion group as compared to that in patent IRA group (11.96 fL vrs $10.92 \mathrm{fL}, \mathrm{p}<0.001$, Table 1). However, there was no significant difference in other blood parameters such as hemoglobin level, blood sugar level, platelets count, PT, APTT, WBC count, serum creatinine, cystatin $\mathrm{C}$ and uric acid between the two study groups (Table 1). Similarly, serum electrolytes such as sodium, potassium, calcium and the parameters of liver function such AST, ALT, serum albumin and total protein, were also compared between two groups, the difference was non-significant. (Table1).

\section{Cardiac biomarkers}

CK-MB was significantly higher in total occlusion group as compared to the patent IRA group (290.2 vs. 190.98, $\mathrm{p}<0.001$, Table 1) however, there was no significant difference found in other biomarker Troponin I between the two groups (14.62 vs. $12.66, \mathrm{p}=0.34$, Table 1 ).

\section{Inflammatory and lipid biomarkers}

Patients in total occlusion group had higher Lp (a) levels as compared to that in patients with patent IRA group ( $179.57 \mathrm{nmol} / 1$ vs $141.16 \mathrm{nmol} / 1, \mathrm{p}<0.001$, Table 1$)$. Total cholesterol level in total occlusion group $(4.11 \mathrm{mmol} / \mathrm{L})$ was higher as compared to that in the patent IRA group (3.8 $\mathrm{mmol} / \mathrm{L}$ ), this difference was also statistically significant ( $\mathrm{p}$ $=0.02$, Table 1). Difference between other parameters such as hs-CRP, HDL, LDL, TG and homocysteine levels were statistically non significance $(\mathrm{p}>0.05$, Table 1$)$

\section{Echocardiography and hospital stay}

Prevalence of regional wall motion abnormality in echocardiogram was significantly higher in total occlusion group as compared to that in patent IRA group $(77.2 \%$ vs $54.2 \%, \mathrm{p}<0.001$, Table 1 and Fig. $1)$, other echocardiography findings were similar between two groups ( $p>0.05$, Table 1). Patients with total occlusion of infarct related coronary artery had longer hospital stay duration as compared to that of patients with patent IRA (13.67 days vs. 11.43 days, $\mathrm{p}<0.01$, Table 1 ).

\section{Discussion}

We compared various blood parameters, such hemoglobin level, blood sugar level, WBC, platelets count, MPV, PT and APTT. Among them MPV level was higher in total occlusion group than that in the patent IRA group; there was statistically significant difference noted between the two group means as $\mathrm{p}$ value was less than 0.05 . Platelets play an important role in the pathogenesis of CAD; MPV, a measure of platelet size, correlates with their reactivity., ${ }^{4,5}$ Orhan Maden et al. evaluated relationship of hematological indexes at the time of admission with myocardial reperfusion abnormalities in acute ST 
Table 1 Descriptive characteristic of two study groups

\begin{tabular}{|c|c|c|c|}
\hline & Total occlusion & Patent IRA & $\mathrm{p}$ value \\
\hline $\mathrm{N}$ & 203 & 84 & \\
\hline Age & 57.59 & 59 & 0.664 \\
\hline $\begin{array}{l}\text { Gender } \\
\text { Male } \\
\text { Female }\end{array}$ & $\begin{array}{c}169(83.25 \%) \\
34(16.75 \%)\end{array}$ & $\begin{array}{l}68(80.95 \%) \\
16(19.05 \%)\end{array}$ & 0.09 \\
\hline Hypertension & $83(40.9 \%)$ & $37(44 \%)$ & 0.34 \\
\hline Diabetes & $24(11.8 \%)$ & $13(15.5 \%)$ & 0.44 \\
\hline Smoking & $133(65.5 \%)$ & $52(61.9 \%)$ & 0.56 \\
\hline Dyslipidemia & $29(14.3 \%)$ & $2(2.4 \%)$ & $0.001^{*}$ \\
\hline Family history of CAD & $16(7.9 \%)$ & $7(8.3 \%)$ & 0.50 \\
\hline Blood sugar (mmol/L) & $8.05 \pm 1.91$ & $7.95 \pm 1.85$ & 0.57 \\
\hline WBC( 109 cells/L) & $8.7 \pm 0.89$ & $8.4 \pm 1.01$ & 0.09 \\
\hline Hemoglobin(g/dl) & $136.62 \pm 36.25$ & $140.43 \pm 13.33$ & 0.384 \\
\hline Platelets & $164.92 \pm 41.31$ & $157.16 \pm 43.59$ & 0.704 \\
\hline MPV & $11.96 \pm 1.23$ & $10.92 \pm 1.13$ & $0.00008^{*}$ \\
\hline APTT & $32.37 \pm 3.50$ & $39.01 \pm 3.61$ & 0.36 \\
\hline PT & $12.85 \pm 0.598$ & $13.2 \pm 0.398$ & 0.481 \\
\hline Uric acid $(\mu \mathrm{mol} / \mathrm{L})$ & $355.03 \pm 94.65$ & $282.84 \pm 68.96$ & 0.68 \\
\hline Cystin C (mg/L) & $0.92 \pm 0.2$ & $0.87 \pm 0.1$ & 0.19 \\
\hline Creatinine $(\mu \mathrm{mol} / \mathrm{L})$ & $90.57 \pm 13.55$ & $78.77 \pm 28.84$ & 0.17 \\
\hline AST (units/L) & $84.85 \pm 13.86$ & $90.61 \pm 20.1$ & 0.24 \\
\hline ALT (units/L) & $45.35 \pm 24.74$ & $49.51 \pm 31.51$ & 0.21 \\
\hline Total protein & $62.69 \pm 5.13$ & $66.10 \pm 7.48$ & 0.22 \\
\hline Serum albumin & $39.45 \pm 2.45$ & $39.1 \pm 3.85$ & 0.62 \\
\hline Sodium & $141.30 \pm 3.45$ & $139.54 \pm 1.37$ & 0.306 \\
\hline Potassium & $3.8 \pm 0.4$ & $4.03 \pm 0.26$ & 0.35 \\
\hline Calcium & $2.27 \pm 0.12$ & $2.3 \pm 0.38$ & 0.304 \\
\hline CK-MB & $290.20 \pm 108.28$ & $190.98 \pm 109.62$ & $0.00014^{*}$ \\
\hline Troponin I & $14.62 \pm 20.83$ & $12.66 \pm 16.09$ & 0.34 \\
\hline hs-CRP & $25.8 \pm 10.4$ & $30.35 \pm 14.6$ & 0.212 \\
\hline Homocysteine & $30.66 \pm 10.17$ & $27.06 \pm 10.17$ & 0.481 \\
\hline $\mathrm{Lp}(\mathrm{a})$ & $179.57 \pm 80.59$ & $141.16 \pm 49.24$ & $0.0003^{*}$ \\
\hline LDL & $2.46 \pm 0.78$ & $2.39 \pm 0.48$ & 0.507 \\
\hline HDL & $0.99 \pm 1.15$ & $1.11 \pm 0.40$ & 0.62 \\
\hline Total Cholesterol & $4.11 \pm 1.17$ & $3.8 \pm 0.57$ & $0.02^{*}$ \\
\hline TG & $2.02 \pm 1.01$ & $1.53 \pm 0.66$ & 0.787 \\
\hline $\mathrm{EF}$ (mean) (in \%) & $52.03 \pm 8.9$ & $53.79 \pm 9.2$ & 0.16 \\
\hline LVID(mean) (in mm) & $53.29 \pm 5.23$ & $52.38 \pm 5.51$ & 0.507 \\
\hline Wall motion abnormality & $156(77.2 \%)$ & $45(54.2 \%)$ & $0.00012^{*}$ \\
\hline
\end{tabular}

Values are expressed as raw data and mean $\pm S D$

${ }^{*} p<0.005$ compared between two groups

N number of patients, MPV mean platelet volume, APTT activated partial thromboplastin time, PT prothrombin time, AST Aspartate transaminase, $A L T$ alanine transaminase, $C K-M B$ creatine kinase- $M B$, hs-CRPhigh sensitive $C$-reactive protein, Lp(a) lipoprotein(a) , LDL low density lipoprotein, HDL high density lipoprotein, TG triglycerides, EF ejection fraction, LVID left ventricular internal dimension. 
segment elevation myocardial infarction patients treated with primary PCI, in which, MPV was found to be independently associated with impaired reperfusion of infarct related artery. ${ }^{6}$ Huczek $Z$ also reported that MPV on admission predicts impaired reperfusion and longterm mortality in acute myocardial infarction treated with primary PCI. ${ }^{7}$ Rodrigo Estévez-Loureiro et al. also reported that MPV predicts patency of the IRA in STEMI before primary PCI, as MPV was significantly higher in total occlusion group as compared to patent IRA group ( $\mathrm{p}$ value $<0.0001) .{ }^{8} \mathrm{MPV}$ level significantly higher in many studies including ours may be due to the role of platelets in pathogenesis of STEMI, as hyperactive platelets is an important determinant of the thrombogenic process of total coronary occlusion of IRA. ${ }^{8}$ Parameters related to kidney function such as serum creatinine, cystatin $\mathrm{C}$ and uric acid were also evaluated in our study. These markers didn't show any significant association with any of the two study group, as $\mathrm{T}$ test done showed $\mathrm{p}$ value $>0.05$ in all these parameters.

Among inflammatory markers and lipid parameters evaluated between our study groups hs-CRP, LDL, HDL, homocysteine and TG, showed no statistically significant difference between the two study groups. A study done by Jin Won Kim et al. showed no significant difference in lipid parameters and hs-CRP between two study groups ( TIMI flow 0 or 1 and TIMI flow 2 or 3 ), as p value was more than 0.05 for HDL, LDL, TG and hs-CRP. ${ }^{9}$ In our study, Lp(a) was shown to have significant effect on the patency of the IRA, as there was statistically significant difference in the mean value of $\mathrm{Lp}$ (a) between the two study groups. This result was again similar to that in above mentioned study done by Jin Won Kim et al., in which, $\mathrm{Lp}$ (a) level was significantly high in total occlusion group as compared to other groups, with $\mathrm{p}$ value less than 0.05 . Similarly, according to Dagdelen's study the spontaneous reperfusion rate was significantly higher in patients with low levels of $\mathrm{Lp}(\mathrm{a})$ within first week of myocardial infarction. ${ }^{10}$ Role of $\mathrm{Lp}(\mathrm{a})$ in pathogenesis of atherosclerosis and myocardial infarction is still not fully understood. As $\mathrm{Lp}$ (a) interacts with cellular matrix protein via apo(a) which may interfere with plasminogen activation resulting either in delayed lysis of mural thrombi, hence contributing to the initiation and progression of atherosclerosis, or in enhanced thrombosis following plaque rupture. This may be the reason for higher level of $\mathrm{Lp}$ (a) levels in total occlusion group in our study. Serum cholesterol level was also found to be in higher level in total occlusion group, which is statistically significant as $\mathrm{p}$ value was less than 0.05 . There was no study supporting this result directly, however, different studies have reported that using cholesterol lowering drug have shown to enhance the patency of IRA in myocardial infarction. ${ }^{11}$

Our study has few limitation, it was a retrospective, single centre study.

\section{Conclusion}

Patients with lower levels of MPV, Lp(a), total cholesterol level and CK-MB at the time of presentation, may have higher chance of having patent IRA in Coronary angiogram and may play some role in predicting patency of IRA in acute ST elevation Myocardial Infarction. However, a multi centered, prospective study is required to further analyze the markers affecting the patency of IRA before we can make any decision on its implication on the preventive and therapeutic measures.

\section{References}

1. John F. Beltrame, Rachel Dreyer and Rosanna Tavella (2012). Epidemiology of Coronary Artery Disease, Coronary Artery Disease - Current Concepts in Epidemiology, Pathophysiology, Diagnostics and Treatment, Dr. David Gaze (Ed.), ISBN: 978-953-51-0262-5, InTech.

2. Van de Werf F, Bax J, Betriu A, et al. Management of acute myocardial infarction in patients presenting with persistent ST-segment elevation: the Task Force on the Management of STSegment Elevation Acute Myocardial Infarction of the European Society of Cardiology. Eur Heart J 2008;29(23):2909-45.

3. Stone GW, Cox D, Garcia E, et al. Normal flow (TIMI-3) before mechanical reperfusion therapy is an independent determinant of survival in acute myocardial infarction: analysis from the primary angioplasty in myocardial infarction trials. Circulation 2001; 104(6):636-41.

4. Thompson CB, Jakubowski JA, Quinn PG, et al. Platelet size and age determine platelet function independently. Blood 1984;63(6):1372-5.

5. Frossard M, Fuchs I, Leitner JM, et al. Platelet function predicts myocardial damage in patients with acute myocardial infarction. Circulation 2004;110(11):1392-7.

6. Maden O, Kacmaz F, Selcuk H, et al. Relationship of admission hematological indexes with myocardial reperfusion abnormalities in acute $\mathrm{ST}$ segment elevation myocardial infarction patients treated with primary percutaneous coronary interventions. Can J Cardiol 2009;25(6):e164-8.

7. Huczek Z, Kochman J, Filipiak KJ, et al. Mean platelet volume on admission predicts impaired reperfusion and long-term mortality in acute myocardial infarction treated with primary percutaneous coronary intervention. J Am Coll Cardiol 2005;46(2):284-90.

8. Estévez-Loureiro R, Salgado-Fernández J, Marzoa-Rivas R, et al. Mean platelet volume predicts patency of the infarct-related artery before mechanical reperfusion and short-term mortality in patients with ST-segment elevation myocardial infarction undergoing primary percutaneous coronary intervention. Thromb Res 2009; 124(5):536-40.

9. Kim JW, Seo HS, Suh SY, et al. Relationship 
between lipoprotein(a) and spontaneous recanalization of infarct-related arteries in the early phase of acute myocardial infarction. Clin Cardiol 2008;31(5):211-6.

10. Dagdelen, S · Eren, N · Akdemir et al. The effect of lipoprotein-a on thrombolytic therapy and spontaneous reperfusion in acute myocardial infarction. A comparative study. Int Angiol.
2002; 21(4):384-9.

11. Takayama M, Munakata K, Tomita Y, et al. 1006-45 Regression of Infarct-Related Coronary Lesions on Aggressive Lipid Lowering Treatment in Patients After Recovery from Acute Myocardial Infarction. J Am Coll Cardiol. 1995;25:357A-357A.
Cite this article as: Reeju Manadhar, Chaofeng Sun. Association of different blood parameters with the early patency of IRA in STEMI. Nepalese Heart Journal 2015;12(2):73-77. 